\title{
Provision of Removable Partial Dentures
}

Determining 'need' for a Removable Partial Denture: a qualitative study of factors that influence dentist provision and patient use

R. Graham, S. Mihaylov, N. Jepson, P. F. Allen and S. Bond Br Dent J 2006; 200: 155-158

Objective

To identify factors that influence Removable Partial Denture (RPD)

provision, and patient use of RPDs in the UK.

Design

Exploratory qualitative interview study.

Subjects and methods

Subjects There were two sample groups. A purposive sample of 16 male and female dentists was categorised in terms of level of RPD provision, experience, and practice characteristics. A purposive sample of 17 male and female partially dentate patients was categorised in terms of RPD use and demographic characteristics. Data collection Semi-structured in-depth interviews.

Results

For dentists, RPD provision was indicated by patient demand and physical function of the remaining teeth, but was mediated by NHS fee structures and professional satisfaction. For patients, RPD use was influenced by the trade-off between improved appearance and the unpalatable presence of an RPD in their mouth. The location of the gap(s) was important, but other issues were relevant such as ability to 'manage' without the RPD.

Conclusion

When defining 'need' for an RPD, dentists focused on physical function of the teeth whereas patients focused on social meanings of the mouth.

These differing priorities may improve understandings of patient noncompliance in RPD use. Further research on the relationship between denture use and social identity could be beneficial.

\section{IN BRIEF}

- Existing research suggests that $30-50 \%$ of patients who are prescribed an RPD never or only occasionally wear the prosthesis.

- This study has identified key factors that influence professional provision and patient use of RPDs.

- For patients, wearing an RPD is not simply a matter of aesthetics, but of avoiding the social stigma associated with tooth loss.

\section{COMMENT}

The purpose of this paper was to identify factors that influence dentists' provision of, and patients' use of, removable partial dentures (RPDs). A purposive sampling strategy was used to identify 1) a sample group of NHS dentists (taking into account frequency of RPD prescription, experience, practice location, population density, and dentist/population ratio) and 2) a sample of NHS patients who had been provided with RPDs (taking account of the number and distribution of the remaining natural teeth and denture wearing habits). Both samples provided the range of opinion that exists within dentist and patient populations. Data, from semi-structured, in-depth interviews, were analysed to identify emerging themes.

The results showed that dentists' decisions about whether to provide RPDs were usually initiated by the patient. This was more likely if an upper or anterior tooth was missing. Where posterior, lower teeth were missing, dentists only recommended RPDs where physical function was affected. Decisions between RPDs and fixed bridgework were primarily influenced by oral hygiene, with the provision of the latter being more likely where mouths were considered 'clean'. Patients with 'dirty' mouths were more likely to be provided with acrylic resin based RPDs. Cost-effectiveness was the other major driver for dentist decision between RPD and fixed bridgework, and between acrylic resin and cobalt-chromium based RPDs.

For patients, the dominant influence on RPD use was aesthetic improvement. Patients were more likely to wear their RPD if: it filled an upper anterior gap obvious to others; if in regular contact with other people; or if the RPD improved the shape of their face/smile. For most patients, physical function was of secondary importance. For some, denture difficulties related to eating, taste, speech and retention outweighed potential aesthetic benefits resulting in nonwearing of the RPD. Dentists providing NHS RPDs are primarily led to do so by patient demand and physical function. For patients, the two key issues are physical function of the entire mouth (not just the teeth) and concerns about appearance.

This well-balanced paper is driven by the desire to provide an evidence-base for the cost-efficient assessment for the provision of RPDs, which currently costs the NHS $£ 50$ million per annum. It demonstrates disparity between dentists' and patients' thinking on the subject. As patient factors are the most important motivators for wearing the RPD, dentists need to consider carefully how appropriate it is to expect a person to wear an RPD to replace posterior teeth for functional reasons when their driver centres on appearance and acceptability. The paper achieves its aim by giving some insight into how the practitioner might explore these issues further with patients before recommending RPDs.

For me the authors left two issues unresolved. Firstly that more consideration could be given to the role of the shortened dental arch; and secondly the debate centring on the dentists' perception that putting RPDs in a dirty mouth does less damage than placing fixed bridgework.

J. Fiske, Senior Lecturer and Consultant in Special Care Dentistry, GKT Dental Institute of King's College London doi: 10.1038/sj.bdj.4813194 